Provided for non-commercial research and education use. Not for reproduction, distribution or commercial use.

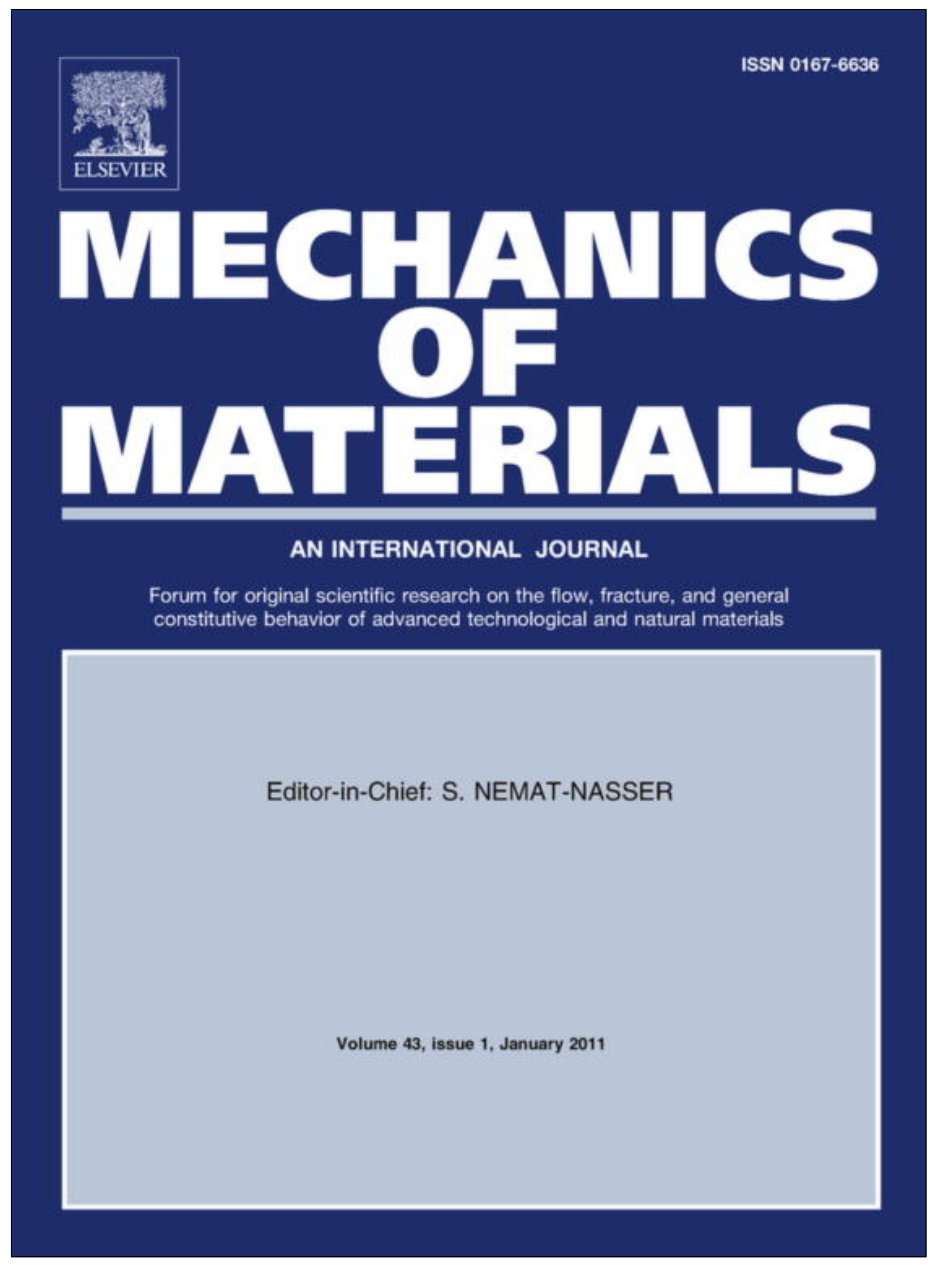

(This is a sample cover image for this issue. The actual cover is not yet available at this time.)

This article appeared in a journal published by Elsevier. The attached copy is furnished to the author for internal non-commercial research and education use, including for instruction at the authors institution and sharing with colleagues.

Other uses, including reproduction and distribution, or selling or licensing copies, or posting to personal, institutional or third party websites are prohibited.

In most cases authors are permitted to post their version of the article (e.g. in Word or Tex form) to their personal website or institutional repository. Authors requiring further information regarding Elsevier's archiving and manuscript policies are encouraged to visit:

http://www.elsevier.com/copyright 


\title{
Visco-elastic behavior through fractional calculus: An easier method for best fitting experimental results
}

\author{
M. Di Paola, A. Pirrotta*, A. Valenza \\ Dipartimento di Ingegneria Civile, Ambientale e Aerospaziale (DICA), Università di Palermo, Viale delle Scienze, 90128 Palermo, Italy
}

\section{A R T I C L E I N F O}

\section{Article history:}

Received 18 March 2011

Received in revised form 8 August 2011

Available online 7 September 2011

\section{Keywords:}

Fractional calculus

Visco-elastic

Relaxation function

Creep function

Power law

\begin{abstract}
A B S T R A C T
In capturing visco-elastic behavior, experimental tests play a fundamental rule, since they allow to build up theoretical constitutive laws very useful for simulating their own behavior. The main challenge is representing the visco-elastic materials through simple models, in order to spread their use. However, the wide used models for capturing both relaxation and creep tests are combinations of simple models as Maxwell and/or Kelvin, that depend on several parameters for fitting both creep and relaxation tests. This paper, following Nutting and Gemant idea of fitting experimental data through a power law function, aims at stressing the validity of fractional model. In fact, as soon as relaxation test is well fitted by power law decay then the fractional constitutive law involving Caputo's derivative directly appears. It will be shown that fractional model is proper for studying visco-elastic behavior, since it may capture both relaxation and creep tests, requiring the identification of two parameters only. This consideration is assessed by the good agreement between experimental tests on creep and relaxation and the fractional model proposed. Experimental tests, here reported are performed on two polymers having different chemical physical properties such that the fractional model may cover a wide range of visco-elastic behavior.
\end{abstract} (c) 2011 Elsevier Ltd. All rights reserved.

\section{Introduction}

The early works dealing with the viscous flow phenomenon have been done in the 19th century, focusing the attention on materials such as metal and glass which do not show marked visco-elastic features under standard or design conditions. In the second half of the 20th century, with the diffusion of the synthetic polymers, the viscoelasticity has been investigated more extensively. In particular at the beginning of the twentieth century, Nutting (1921) and Gemant (1936) observed that, for visco-elastic materials such as rubber, bitumen, polymers, concrete etc., the experimental data were well fitted by a power law decay. Later on it has been shown that the transfer function of visco-elastic materials may be identified in frequency domain by means of real powers of frequency leading, by inverse Fourier transform, to fractional operators in

\footnotetext{
* Corresponding author.

E-mail address: antonina.pirrotta@unipa.it (A. Pirrotta).
}

time domain (Scott-Blair and Gaffyn, 1949). In particular, the power law decay representation for relaxation data was firstly considered by the polymer scientists, while the mechanics community chose the classical models as the Maxwell model, the Kelvin-Voight model and complex combinations of these elementary models to capture viscoelastic phenomena (Flugge, 1967; Christensen, 1982; Pipkin, 1972). The main problems arising from these combinations of "classical" models are: (i) to reproduce the actual material visco-elastic behavior (creep and relaxation phases) many parameters have to be set leading to possible high computational effort, (ii) the parameters are found by means of best fitting numerical procedure which can lead to meaningless parameters from physical point of view (e.g. negative coefficients of stiffness in a spring and of viscosity of dashpots), (iii) the constitutive laws of such models are always differential equations with integer order of derivation whose solutions are in exponential form. As a consequence, the response in terms of creep or relaxation is exponential or linear. 
If the power law decay is considered for relaxation function, a different kind of linear operator is needed for the analysis as the fractional operator that is a differential one of real order. As a consequence the constitutive law is ruled by a fractional differential equation. Fractional calculus can be regarded as an extension of the classical differential calculus. Until now a limited use of such tool can be observed in mechanics, probably due to the presence of many definitions of fractional operators as well as the lack of an easy geometrical meaning. However, the common point to all the definitions of fractional operators is that they are simply convolution integrals with power law kernel. The beauty of such operators is that they exactly behave as ordinary derivatives and integrals, that is all the rules of classical operators with integer order hold true, including Leibniz rule and integration by parts. Moreover also in Fourier and in Laplace domain the rules are quite similar and simple like the case of the classical derivatives and integrals of integer order (Scott-Blair and Gaffyn, 1949; Hilfer, 2000; Samko et al., 1993).

In this regard, the fractional constitutive law, viewed as a generalization of the classical visco-elastic constitutive law, serves to bring several concepts of visco-elastic modeling into one coherent approach having a foundation in molecular theory (Gonsovski and Rossikhin, 1973; Stiassnie, 1979; Mainardi and Gorenflo, 2007).

In this paper it is shown that if relaxation test is well fitted by a power law decay then the fractional constitutive law involving Caputo's derivative directly appears. Such a constitutive law is defined by only two parameters, getting rid from the use of combinations of simple models dependent on several parameters to capture both creep and relaxation tests.

On the other hand, since creep and relaxation functions are linked in Laplace domain, then from a theoretical point of view only one test is enough to determine the relevant parameters of the visco-elastic behavior. Usually, in literature, only one experimental test is performed (creep or relaxation). Here, in order to fully validate the fractional model, two polymers of different chemical physical properties are tested. For each of them both relaxation and creep test is performed. The best fitting for the identification of visco-elastic parameters is developed by using one of the two experimental curves, with these parameters the other theoretical function is readily found and the analytical curve is compared with the correspondent experimental one. Tests are performed with different values of amplitudes and always the theoretical relaxation and creep functions overlap the experimental data leading to the conclusion that the fractional model is able to fully capture the visco-elastic behavior, covering a wide range of behavior (for instance the two materials used in the tests have different mechanical characteristic one from each other).

\section{Visco-elastic behavior}

For a linear elastic helical spring, the stress is always directly proportional to the corresponding strain in small deformations but it is independent of strain-rate. During the deformation process mechanical energy is stored with no dissipation, so that when the external load is removed, the helical spring returns to its original length. On the other hand in a system composed of a piston with perforated bottom, moving inside a cylinder filled with an ideal viscous fluid (dashpot), the stress is directly proportional to the strain-rate, but independent of the strain itself. Such an ideal system, in fact, exhibits a capacity for entirely dissipating energy but none for storing it. Real materials exhibit a mixture of the two simple behaviors and then are called visco-elastic ones.

Such a material does not maintain a constant strain under constant stress, but it undergoes strain slowly varying with time, that is, it creeps; and if deformed at constant strain, the stress required to hold it diminishes gradually with time, that is, it relaxes.

Then, it is apparent that, in order to study the viscoelastic material under an assigned forcing function, we need tests to capture both creep and relaxation phases. Regarding the relaxation phase, the test is performed, applying a constant strain $\varepsilon_{0},(\forall t \geqslant 0)$ and measuring the corresponding stress $\sigma(t)$. If we assume that the system is linear, then the stress will be proportional to $\varepsilon_{0}$ through the so called relaxation function $E(t)$, that is independent of the imposed strain $\varepsilon_{0}$. Then for a linear visco-elastic model we write:

$\sigma(t)=E(t) \varepsilon_{0} ; \quad \forall t \geq 0$.

Relaxation function $E(t)$ is the stress history per unit strain imposed. Conversely, as soon as we are concerned with creep phase, it suffices to invert the procedure, applying a constant stress $\sigma_{0},(\forall t \geqslant 0)$, and measuring the time dependent strain $\varepsilon(t)$. Again, since the linear behavior, the strain will be proportional to $\sigma_{0}$ through the so called creep function $D(t)$ (independent of the imposed stress $\sigma_{0}$ ):

$\varepsilon(t)=D(t) \sigma_{0} ; \quad \forall t \geq 0$.

Creep function $D(t)$ is the strain history per unit stress imposed.

The two functions are not independent of each other, since they are connected by a simple relation (Scott-Blair and Gaffyn, 1949):

$D(s) E(s)=s^{-2}$,

where $D(s)$ and $E(s)$ are the Laplace transforms of $D(t)$ and $E(t)$, respectively.

The uniaxial, isothermal stress-strain equation for a linear visco-elastic material is ruled by the Boltzman superposition integral:

$\sigma(t)=\int_{0}^{t} E(t-\bar{t}) \frac{d \varepsilon(\bar{t})}{d \bar{t}} d \bar{t}$

That, in this form, is valid for a quiescent system at $(t=0)$. The right-hand side of the Eq. (4) is a convolution integral in which the relaxation function $E(t)$ plays the role of kernel of the integral in Eq. (4). From experimental data it is always observed that $E(t)$ is a decaying function and the shape of such decaying function depends on the material.

The simplest model of $E(t)$ is an exponential decay in the form:

$E(t)=E \exp (-\alpha t)$, 


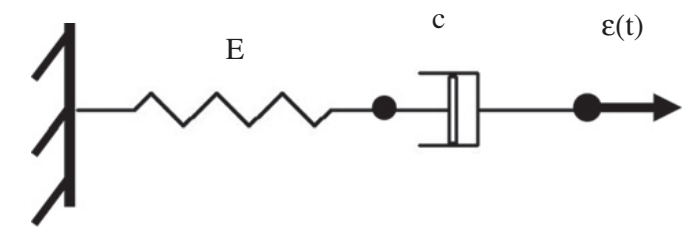

Fig. 1a. Maxwell model.

where $E$ and $\alpha=E / c$ are two parameters obtained from experimental data. This choice leads to the Maxwell unit, depicted in Fig. 1a in which an imposed strain is applied.

By denoting the stress in the spring with $\sigma(t)$, the equation ruling the evolution of the stress for an imposed strain $\varepsilon(t)$ is given as:

$\dot{\sigma}(t)+\alpha \sigma(t)=E \dot{\varepsilon}(t)$.

For the quiescent system at $(t=0)$ the Duhamel integral corresponding to Eq. (6) coalesces with $\sigma(t)$ given in Eq. (4). On the other hand the creep function corresponding to Eq. (5) may be obtained by the inverse Laplace Transform of $E(s)^{-1} s^{-2}$ (according to Eq. (3)):

$D(t)=\frac{1}{E}+\frac{t}{c}$,

that is for the Maxwell unit, corresponding to the exponential (5), the creep function is linear and the behavior does not correspond to any experimental observation of real materials.

The creep phase is better described by the Kelvin Voigt unit depicted in Fig. 1b, in which the spring and the dashpot are in parallel and excited by an assigned stress $\sigma(t)$. The equilibrium equation gives:

$\dot{\varepsilon}(t)+\alpha \varepsilon(t)=\frac{\sigma(t)}{c}$,

that for a quiescent system at $(t=0)$ corresponds to a Duhamel convolution integral:

$\varepsilon(t)=\frac{1}{c} \int_{0}^{t} \exp (-\alpha t-\bar{t}) \sigma(\bar{t}) d \bar{t}$

For such a model the creep function has the form:

$D(t)=\frac{1-\exp (-\alpha t)}{E}$,

and the corresponding relaxation function is a constant.

Then we conclude that since two different models are necessary to describe creep and relaxation, both simple models cannot be used for description of the visco-elastic behavior. In order to fill this physical inconsistency of the

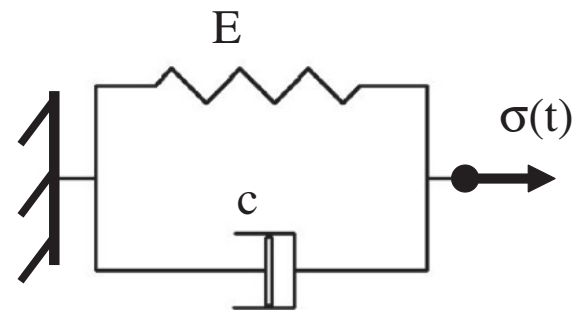

Fig. 1b. Kelvin-Voigt model. two visco-elastic models above described, combinations of Kelvin-Voigt and Maxwell units are often used in order to better fit experimental data (Flugge, 1967). However using such a more sophisticated models involving springs and dashpots, since the solutions remain in the class of exponential functions for creep and relaxation functions, then the latter cannot simultaneously fit experimental data.

In the next section, in order to properly define, both creep and relaxation phases with a very simple model, the fractional model will be introduced.

\section{Fractional model}

At the beginning of the twentieth century, Nutting (1921), Gemant (1936) observed that, for visco-elastic materials such as rubber, bitumen, polymers, concrete etc., the experimental data coming from the relaxation test, were well fitted by a power law decay, that is:

$E(t) \propto(t)^{-\beta}, \quad 0<\beta<1$.

If we select the coefficient of proportionality in Eq. (11) in the form $E \tau^{\beta} \mid \Gamma(1-\beta)$, where $\Gamma(\cdot)$ is the Gamma function, $E \tau^{\beta}=c_{\beta}$ and $\beta$ are coefficients obtained by a best fitting procedure from the relaxation test, then Eq. (11) becomes:

$E(t)=\frac{E}{\Gamma(1-\beta)}\left(\frac{t}{\tau}\right)^{-\beta}=\frac{c_{\beta}}{\Gamma(1-\beta)} t^{-\beta}$

It is worth stressing, that $E$ is the Young modulus $[E]=\mathrm{N} /$ $\mathrm{mm}^{2}$ and $\tau$ is a time $[\tau]=\mathrm{s}$.

Once $E(t)$ is written as in Eq. (12) the Boltzman superposition principle restitutes the constitutive law in the form:

$\sigma(t)=\frac{c_{\beta}}{\Gamma(1-\beta)} \int_{0}^{t}(t-\bar{t})^{-\beta} \frac{d \varepsilon(\bar{t})}{d \bar{t}} d \bar{t}=c_{\beta}\left({ }_{c} D_{0^{+}}^{\beta} \varepsilon\right)(t)$

where $\left({ }_{c} D_{0^{+}}^{\beta} \varepsilon\right)(t)$ is the Caputo's fractional derivative, that is valid for a quiescent system at $t=0$. On the other hand, the Caputo's fractional derivative for a quiescent system at $t=0$ or for systems that operate from $t=-\infty$ coalesces with the Riemann-Liouville ( $R L$ ) fractional derivative and the results in literature operating with RL fractional derivative (and quiescent systems at $t=0$ or $t=-\infty$ ) lead to identical results (Podlubny, 1999).

The remarkable result in Eq. (13) for the constitutive law of a visco-elastic material directly descends in assuming that the kernel in the convolution integral is of power law type (Schiessel and Blumen, 1993; Bagley and Torvik, 1979, 1983a,b, 1986; Evangelatos and Spanos, 2011; Schmidt and Gaul, 2002; Di Paola et al., 2011). Then we may affirm that integral equation with kernel of exponential type leads to ordinary differential equation, while when the kernel is of power law type leads to fractional differential equation. It has to be stressed that the constitutive law in Eq. (13) interpolates the purely elastic behavior $(\beta=0)$ and the purely viscous behavior $(\beta=1)$, and is termed in literature (Samko et al., 1993) as springpot element depicted in Fig. 2.

The Laplace transform of $E(t)$ given in Eq. (12) is given as: $E(s)=c_{\beta} s^{\beta-1}$, 


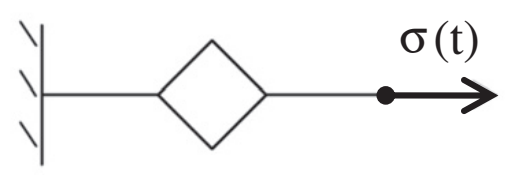

Fig. 2. Springpot element: fractional model.

and then according to Eq. (3), $D(s)=1 /\left(c_{\beta} s^{1+\beta}\right)$ whose inverse Laplace transform restitutes the creep function $D(t)$ in the form:

$D(t)=\frac{1}{c_{\beta} \Gamma(1+\beta)} t^{\beta}=\frac{1}{E \Gamma(1+\beta)}\left(\frac{t}{\tau}\right)^{\beta}$.

Summing up for the definition of the relaxation function $E(t)$ we need to perform a best fitting between the experimental data and the theoretical curve described by Eq. (12), that is we need to define the two parameters $c_{\beta}$ and $\beta$. Once these two parameters are identified the fractional order derivative to describe the constitutive law (13) is derived. On the other hand, because of the Eq. (3), the creep compliance function $D(t)$ is easily derived by Eq. (15). Since no new parameters appear, we conclude that if the experimental test is performed to capture the creep phase and the best fitting is performed to identify $c_{\beta}$ and $\beta$ through the creep test, then also the relaxation function is easily obtained and vice versa.

Now, usually in literature the tests are performed considering creep test only or relaxation test only and the direct connection between the two tests is not evidenced.

In order to fill this gap in the following section the experimental test on two elastomers are performed in both creep and relaxation test. The best fitting is performed to capture $c_{\beta}$ and $\beta$ through relaxation test and with these parameters the theoretical creep function is obtained and compared with the experimental creep data. In this way, we may affirm that the springpot model defined by fractional constitutive law is able to describe in a unique manner both creep and relaxation phases and then it is the correct model for visco-elastic materials.

\section{Experimental tests}

In this section, the experimental tests for two different kinds of elastomers, labeled Aerstop CN 20 and Aerstop VX 5 have been performed, to validate the theoretical results outlined in the previous section. The characteristics of the two elastomers, (provided by Plastitalia Spa of Brolo (ME, Italy)), are reported in the Appendix in Tables A.1 and $A .2$ respectively showing that these materials proper define a wide range of behavior of polymers, having different characteristic one from each other. For each elastomer, tensile, relaxation and creep tests have been performed. From the tensile test, on a proper small-sized specimens thickness $5 \mathrm{~mm}$ (according to Italian standards dealing with plastic materials (UNI EN ISO 527-1 Plastics for tensile properties, and UNI EN ISO 527-2 Plastics for molding and extrusion)), the ultimate stress $\sigma_{u}$ has been derived.
With these data the relaxation and the creep tests have been performed for a small percentage of $\sigma_{u}$.

Tensile experimental results for the two elastomers, are shown in Fig. 3, while in Table 1, it has been reported the value of the ultimate stress $\sigma_{u}\left[\mathrm{~N} / \mathrm{mm}^{2}\right]$, the corresponding strain, and the selected values of the percentage of the ultimate stress with the correspondent strain values.

Moreover, regarding relaxation and creep tests, the strain and the stress correspondent to both of the following values: $10 \% \sigma_{u}, 30 \% \sigma_{u}$, has been considered having fixed the total time duration to $1400 \mathrm{~s}$ that may be considered as sufficiently representative. In more details, in Fig. 4a experimental results of relaxation test on Aerstop CN20, and in Fig. 4b the experimental results of the creep tests are reported. In both figures, the upper curves is pertaining to test with respect to the strain and the stress correspondent to $30 \% \sigma_{u}$, and the other one is dependent on $10 \% \sigma_{u}$. Analogous results are reported in Figs. $5 \mathrm{a}$ and $5 \mathrm{~b}$ for Aerstop VX5.

\section{Comparison between experimental and theoretical results}

According to the power law decay model to fit the experimental data obtained from each relaxation test, we consider the theoretical stress curve, Eq. (1) particularized through Eq. (12), rewritten in the form:

$\sigma(t)=\frac{c_{\beta}}{\Gamma(1-\beta)} t^{-\beta} \varepsilon_{\%}=R t^{-\beta} ; \quad \beta>0$,

having properly relabeled $\varepsilon_{0}$ with $\varepsilon_{\%}$ that is the strain value correspondent to the percentage of the maximum stress $\% \sigma_{u}$ (reported in Table 1 ), considered in performing tests. By looking at Eq. (16) it is apparent that the theoretical

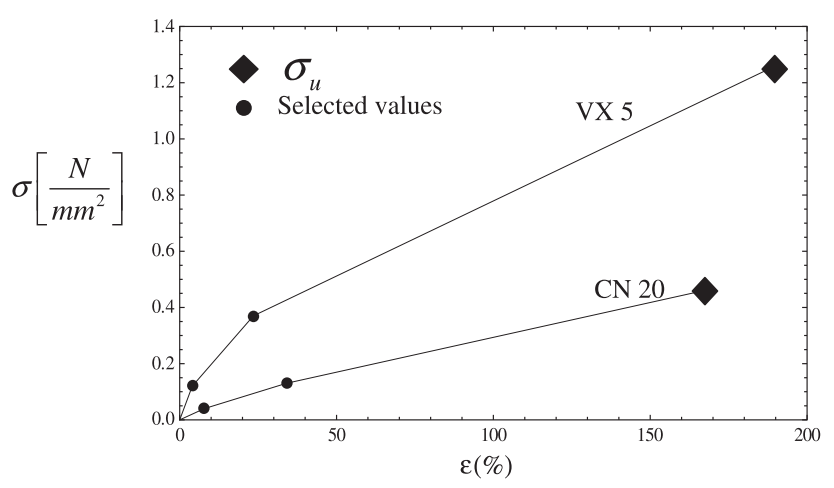

Fig. 3. Tensile experimental results.

Table 1

Strain values at the selected values of the percentage of ultimate stress.

\begin{tabular}{lclc}
\hline & $\sigma_{u}$ & $10 \% \sigma_{u}$ & $30 \% \sigma_{u}$ \\
\hline$C N 20$ & & & \\
$\sigma\left[\mathrm{N} / \mathrm{mm}^{2}\right]$ & 0.461 & 0.04 & 0.13 \\
$\varepsilon[\%]$ & 167 & 7.8 & 34 \\
$V X 5$ & & & \\
$\sigma\left[\mathrm{N} / \mathrm{mm}^{2}\right]$ & 1.26 & 0.12 & 0.37 \\
$\varepsilon[\%]$ & 190 & 4 & 23.5 \\
\hline
\end{tabular}




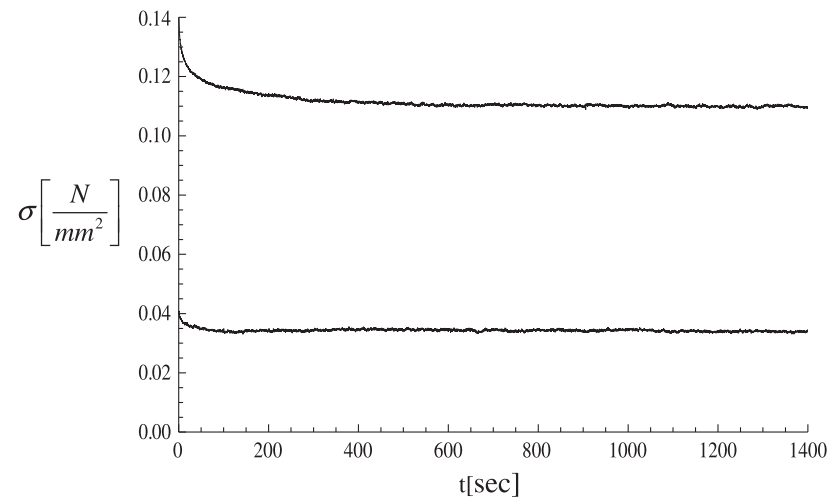

Fig. 4a. Relaxation experimental data on Aerstop CN20.

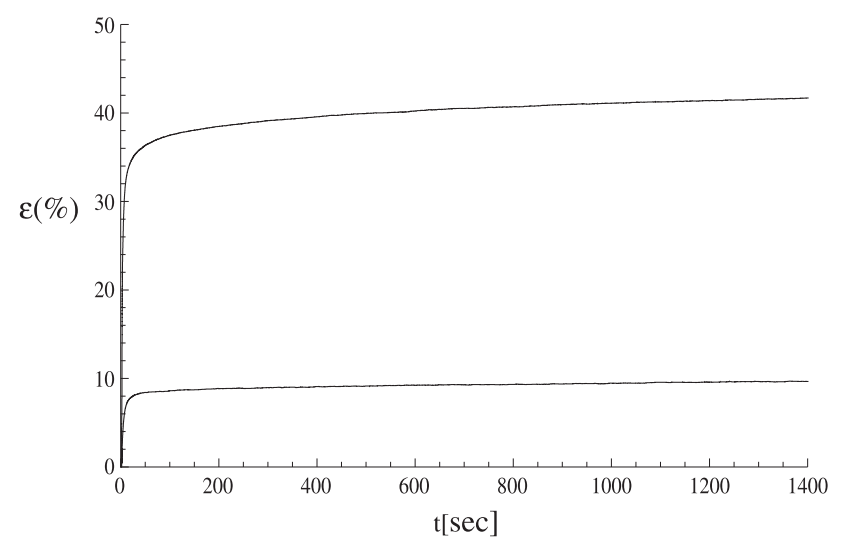

Fig. 4b. Creep experimental data on Aerstop CN20.

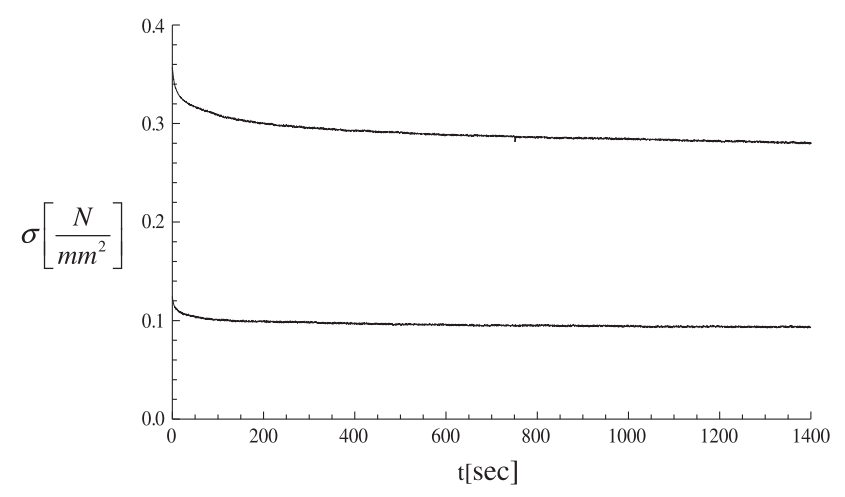

Fig. 5a. Relaxation experimental data on Aerstop VX5.

stress curve is expressed in function of $R$ and $\beta$ that are searched through a best-fitting procedure and reported in Table 2 .

Fig. 6 shows the accuracy of the identified values, since the good agreement between the experimental data and the particularized curve (16), for relaxation test on Aerstop CN20 for both data correspondent to different percentage of maximum stress considered. Analogous results are shown in Fig. 7 for relaxation test on Aerstop VX5 for both data correspondent at different percentage of maximum stress.

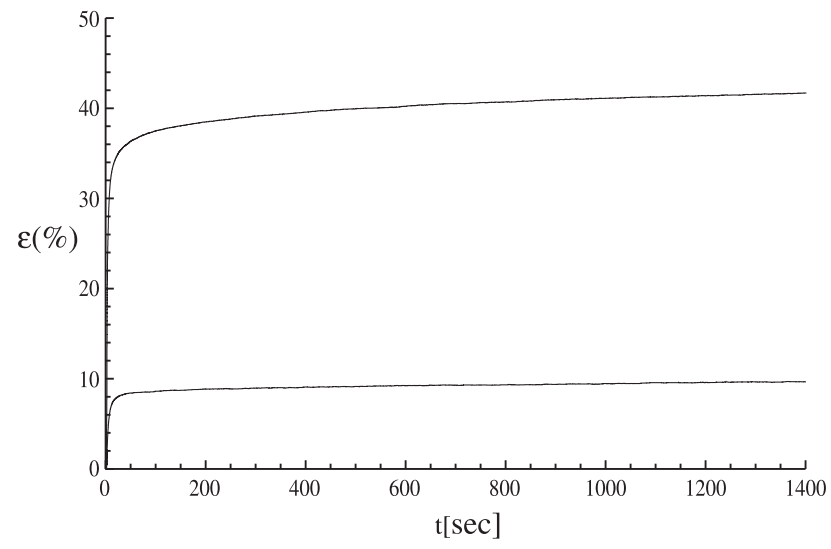

Fig. 5b. Creep experimental data on Aerstop VX5.

Table 2

Identified parameters.

\begin{tabular}{lll}
\hline & $R$ & $\beta$ \\
\hline CN 20 & & \\
$30 \% \sigma_{\mathrm{u}}$ & 0.13 & 0.025 \\
$10 \% \sigma_{\mathrm{u}}$ & 0.04 & 0.025 \\
VX 5 & & \\
$30 \% \sigma_{\mathrm{u}}$ & 0.36 & 0.034 \\
$10 \% \sigma_{\mathrm{u}}$ & 0.12 & 0.034 \\
\hline
\end{tabular}

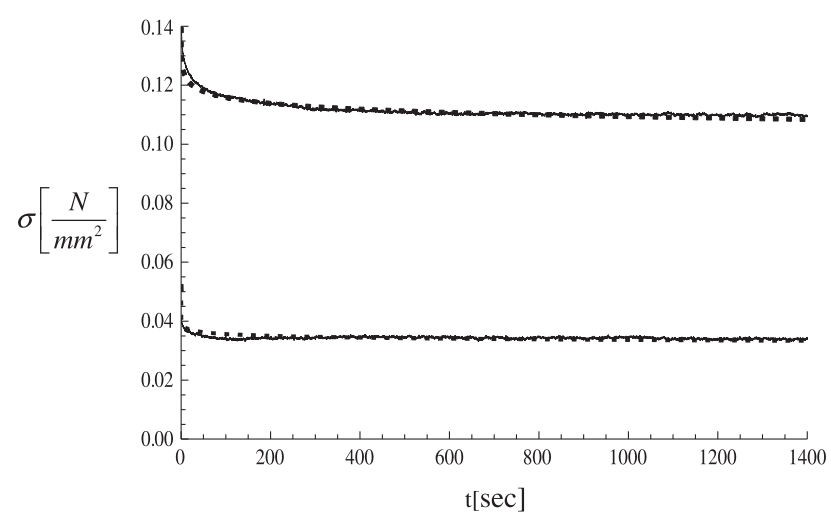

Fig. 6. Relaxation tests on Aerstop CN20: comparison between experimental data and particularized Eq. (16) dotted line.

Further, in order to assess that, through the same fractional model (springpot) it is possible to fit experimental data of both relaxation and creep tests, we first derive the theoretical creep curve with the parameters obtained from the relaxation test and with these parameters we compare the theoretical curve, so obtained, with the experimental one from the creep test. To aim at this, relabeling $\varepsilon_{0}$ and $\sigma_{0}$ with $\varepsilon_{\%}$ and $\sigma_{\%}$, respectively, that are the strain and stress values correspondent to the percentage of the maximum stress $\% \sigma_{u}$ (reported in Table 1 ), considered in performing tests, the creep theoretical curve Eq. (2) assumes the form: 


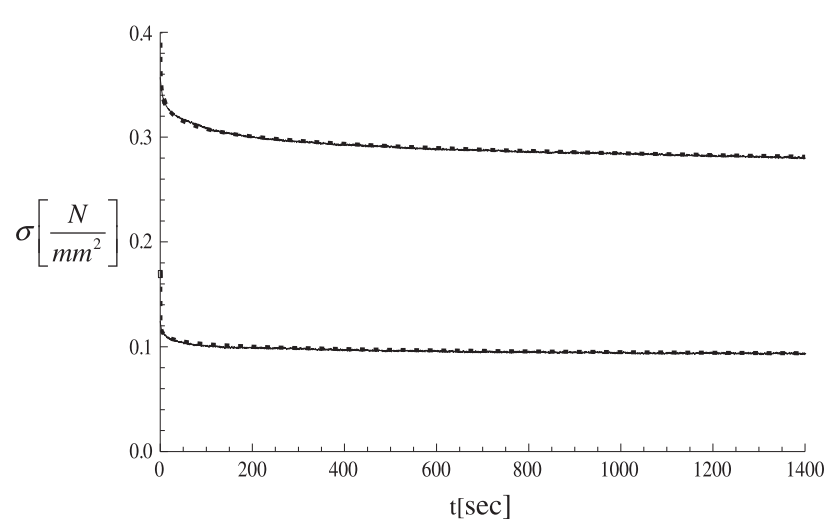

Fig. 7. Relaxation tests on Aerstop VX5: comparison between experimental data and particularized Eq. (16) dotted line.

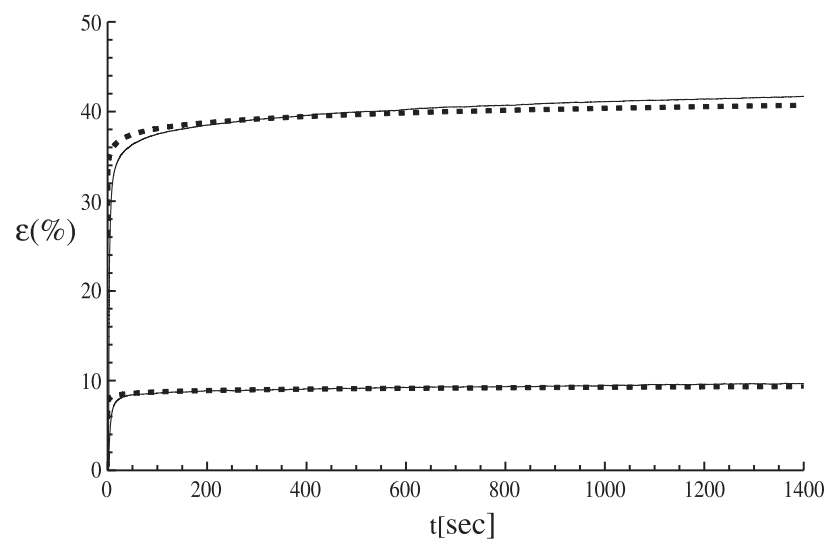

Fig. 8. Creep tests on Aerstop CN20: comparison between experimental data and particularized Eq. (18) dotted line.

$\varepsilon(t)=\frac{1}{c_{\beta} \Gamma(1+\beta)} t^{\beta} \sigma_{\%}$,

having taken into account Eq. (15). Then, considering Eq. (16), leads to the following expressions:

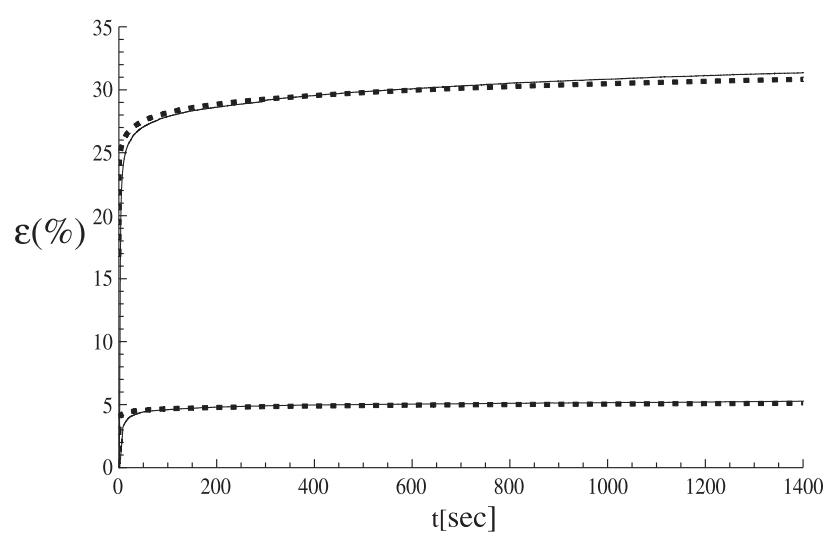

Fig. 9. Creep tests on Aerstop VX5: comparison between experimental data and particularized Eq. (18) dotted line. $\varepsilon(t)=\frac{\sigma_{\%} \varepsilon_{\%}}{R \Gamma(1+\beta) \Gamma(1-\beta)} t^{\beta}$

where all parameters are already known, especially $R$ and $\beta$ from the relaxation experimental data.

It is worth stressing, that curve (18) match quite well the experimental creep data, for both elastomers and for both percentage of maximum stress, assessing the validity of linear fractional visco-elasticity as shown in Figs. 8 and 9. In particular, Fig. 8 is pertaining results on Aerstop CN20 and Fig. 9 shows results on Aerstop VX5.

As conclusive remarks, from the latter figures, it may be evicted the good match between the experimental data and the power law decay Eq. (16) for relaxation test (Figs. 6 and 7) and Eq. (18) for the creep one (Figs. 8 and 9).

This stresses the validity of fractional calculus in formulating the constitutive law for visco-elastic materials, that leads to a fractional model, that allows to capture experimental data of both relaxation and creep tests, by identifying two parameters only, as shown in Figs. 6-9.

\section{Conclusions}

In this paper it is stressed the validity of fractional model to capture visco- elastic behavior. Validity in a wide sense, not only from an accuracy point of view but, especially from the facility of use, being a simple formulation characterized by only two parameters. In fact, it is shown that if relaxation test is well fitted by a power law decay, as Nutting and Gemant proposed at the beginning of the twentieth century, then the fractional constitutive law involving Caputo's derivative directly appears. As a consequence the constitutive law is ruled by a fractional differential equation. Fractional calculus can be regarded as an extension of the classical differential calculus and fractional operators are simply convolution integrals with power law kernel. It is worth of notice, that such a constitutive law can capture both relaxation and creep behavior just identifying only two parameters. This important remark gets rid of the use of combinations of simple models as Maxwell and/or Kelvin, that depend on several parameters for capturing both creep and relaxation tests. Moreover, in order to fully validate the fractional model, two polymers of different chemical physical properties have been tested. For each of them both relaxation and creep test is performed. The best fitting for the identification of the two visco-elastic parameters is developed by using one of the two experimental curve, with these parameters the other theoretical function is readily found and the analytical curve is compared with the correspondent experimental one. Tests are performed with different values of amplitudes and always the theoretical relaxation and creep functions overlap the experimental data leading to the conclusion that the fractional model is able to fully capture the visco-elastic behavior and that may be widely used, since these tests are performed on polymers that have different chemical physical properties. 


\section{Appendix A}

Table A.1

Aerstop CN 22: data sheet.

\begin{tabular}{|c|c|c|c|}
\hline \multirow{2}{*}{$\begin{array}{l}\text { Color: BLACK } \\
\text { Features }\end{array}$} & \multirow{2}{*}{$\begin{array}{l}30 \mathrm{~mm} \\
\text { Method } \quad 70 \mathrm{~mm}\end{array}$} & \multicolumn{2}{|c|}{ CR closed cell expanded rubber } \\
\hline & & Unit & Value \\
\hline Density & ISO $845-88$ & $\mathrm{~kg} / \mathrm{m}^{3}$ & $130 / 170$ \\
\hline Shore hardness 00 & & sh00 & $38-55$ \\
\hline \multirow[t]{2}{*}{ Compression-deflection } & ASTM D 1056-85 -25\% & $\mathrm{kPa}$ & $35 \div 63\left(53^{*}\right)$ \\
\hline & NF R 99211-80 -50\% & $\mathrm{kPa}$ & $80 \div 160\left(133^{*}\right)$ \\
\hline Vacuum-water absorption & ASTM D 1056-80 & $\%$ & $\leqslant 5\left(0.7^{*}\right)$ \\
\hline Compression set & ASTM D $1056-7723^{\circ} \mathrm{C}$ & $\%$ & $\leqslant 25\left(16^{*}\right)$ \\
\hline $50 \% / 22 \mathrm{~h}$ & NF R 99211-80 $40^{\circ} \mathrm{C}$ & $\%$ & $\leqslant 60\left(51^{*}\right)$ \\
\hline Linear shrinkage & After $22 \mathrm{~h} 70{ }^{\circ} \mathrm{C}$ & $\%$ & $<7\left(4.1^{*}\right)$ \\
\hline Ultimate elongation & DIN53571 & $\%$ & $\geqslant 100$ \\
\hline Tensile strength & DIN53571 & $\mathrm{kPa}$ & $\geqslant 500$ \\
\hline Tear resistance & NFR 99211-80 & $\mathrm{kN} / \mathrm{m}$ & $\geqslant 0.5$ \\
\hline
\end{tabular}

* Mean value.

Table A.2

AerstopVX5: data sheet.

\begin{tabular}{|c|c|c|c|}
\hline Color: BLACK & $30 \mathrm{~mm}$ & & NR + SBR closed cell expanded rubber \\
\hline Features & Method & Unit & Value \\
\hline Density & ISO $845-88$ & $\mathrm{~kg} / \mathrm{m}^{3}$ & $350 \pm 50$ \\
\hline Shore hardness 00 & & sh00 & $65 \pm 10$ \\
\hline \multirow[t]{2}{*}{ Compression-deflection } & ASTM D $1056-85-25 \%$ & $\mathrm{kPa}$ & $119 \div 168\left(158^{*}\right)$ \\
\hline & NF R 99211-80 -50\% & $\mathrm{kPa}$ & $300 \div 1000\left(158^{*}\right)$ \\
\hline Vacuum-water absorption & ASTM D 1056-80 & $\%$ & $\leqslant 5\left(0.5^{*}\right)$ \\
\hline Compression set & ASTM D $1056-7723^{\circ} \mathrm{C}$ & $\%$ & $\leqslant 25\left(6^{*}\right)$ \\
\hline $50 \% / 22 \mathrm{~h}$ & NF R 99211-80 $40^{\circ} \mathrm{C}$ & $\%$ & $\leqslant 60\left(22^{*}\right)$ \\
\hline Dimensional stability & After 3 days $80^{\circ} \mathrm{C}$ & $\%$ & $-5\left(-1.2^{*}\right)$ \\
\hline Linear shrinkage & After 7 days $70^{\circ} \mathrm{C}$ & $\%$ & $\max -5\left(-1.6^{*}\right)$ \\
\hline Ultimate elongation & ISO 1798-97 & $\%$ & $\left(220^{*}\right)$ \\
\hline Tensile strength & ISO 1798-97 & $\mathrm{kPa}$ & $\left(1900^{*}\right)$ \\
\hline Tear resistance & ISO 34-1 (B-a) 94 & $\mathrm{kN} / \mathrm{m}$ & $\left(2.2^{*}\right)$ \\
\hline \multirow[t]{2}{*}{ Resistance } & Air + U.V. & & Medium \\
\hline & Oil & & Weak \\
\hline \multirow[t]{2}{*}{ Temperature range } & Constant & ${ }^{\circ} \mathrm{C}$ & $-30+70$ \\
\hline & Intermittent & ${ }^{\circ} \mathrm{C}$ & +80 \\
\hline \multirow[t]{4}{*}{ Specification standards } & ASTM D 1056-77 & RE $45 \mathrm{~B}$ & \\
\hline & AFNOR NF-R 99-211 & $1 \mathrm{C} 30 \mathrm{~B} 4$ & \\
\hline & SAE J 18-79 & RE $45 \mathrm{~B}$ & \\
\hline & FMVSS 302 & Conformable & \\
\hline
\end{tabular}

Mean value.

\section{References}

Bagley, R.L., Torvik, P.J., 1979. A generalized derivative model for an elastomer damper. The Shock and Vibration Bulletin 49, 135-143.

Bagley, R.L., Torvik, P.J., 1983a. A theoretical basis for the application of fractional calculus. Journal of Rheology 27, 201-210.

Bagley, R.L., Torvik, P.J., 1983b. Fractional calculus - a different approach to the analysis of viscoelastically damped structures. American Institute of Aeronautics and Astronautics (AIAA) Journal 20, 741-774.

Bagley, R.L., Torvik, P.J., 1986. On the fractional calculus model of viscoelastic behavior. Journal of Rheology 30 (1), 133-155.

Christensen, R.M., 1982. Theory of Viscoelasticity: An Introduction. Academic Press.

Evangelatos, G.I., Spanos, P.D., 2011. An accelerated newmark scheme for integrating the equation of motion of nonlinear systems comprising restoring elements governed by fractional derivatives. Recent Advances in Mechanics I, 159-177. doi:10.1007/978-94-007-05579_9.
Flugge, W., 1967. Viscoelasticity. Blaisdell Publishing Company, Massachusetts.

Gemant, A., 1936. A method of analyzing experimental results obtained by elasto-viscous bodies. Physics 7, 311-317.

Gonsovski, V.L., Rossikhin, Y.u.A., 1973. Stress waves in a viscoelastic medium with a singular hereditary kernel. Journal of Applied Mechanics and Technical Physics 14 (4), 595-597.

Hilfer, R., 2000. Applications of Fractional Calculus in Physics. World Scientific, Singapore.

Mainardi, F., Gorenflo, R., 2007. Time-fractional derivatives in relaxation processes: a tutorial survey. Fractional Calculus and Applied Analysis 10 (3), 269-308.

Nutting, P.G., 1921. A new general law deformation. Journal of the Franklin Institute 191, 678-685.

Di Paola, M., Failla, G., Pirrotta, A., 2011. Stationary and non-stationary stochastic response of linear fractional viscoelastic systems. Probabilistic Engineering Mechanics in press. doi:10.1016/ j.probengmech.2011.08.017. 
Pipkin, A., 1972. Lectures on viscoelasticity theory. Applied Mathematical Sciences. Springer-Verlag.

Podlubny, I., 1999. On Solving Fractional Differential Equations by Mathematics. Science and Engineering, vol. 198. Academic Press.

Samko, G.S., Kilbas, A.A., Marichev, O.I., 1993. Fractional Integrals and Derivatives. Gordon and Breach, Amsterdam.

Schiessel, H., Blumen, A., 1993. Hierarchical analogues to fractional relaxation equations. Journal of Physics A 26, 5057-5069.
Schmidt, A., Gaul, L., 2002. Finite element formulation of viscoelastic constitutive equations using fractional time derivatives. Non linear Dynamics 29, 37-55.

Scott-Blair, G.W., Gaffyn, J.E., 1949. An application of the theory of quasiproperties to the treatment of anomalous strain-stress relations. The Philosophical Magazine 40, 80-94.

Stiassnie, M., 1979. On the application of fractional calculus on the formulation of viscoelastic models. Applied Mathematical Modelling 3, 300-302. 